\title{
An Implementation of Indoor Location Detection Systems Based on Identifying Codes
}

\author{
Rachanee Ungrangsi ${ }^{1}$, Ari Trachtenberg ${ }^{2}$, and David Starobinski ${ }^{2}$ \\ 1 Computer Science Program, Shinawatra University, \\ Pathumtani 12160, Thailand \\ rachanee@shinawatra.ac.th \\ 2 Electrical and Computer Engineering Department, \\ Boston University, MA 02215, USA \\ $\{$ trachten, staro\}@bu.edu
}

\begin{abstract}
We present the design, implementation and evaluation of a location detection system built over a Radio Frequency network based on the IEEE 802.11 standard. Our system employs beacons to broadcast identifying packets from strategic positions within a building infrastructure in such a way that each resolvable position is covered by a unique collection of beacons; a user of such a system can thus determine his location by means of the beacon packets received. The locations from which beacons broadcast is determined from a formalization of the problem based on identifying codes over arbitrary graphs. We present experimental evidence that our location detecting system is practical and useful, and that it can achieve good accuracy even with a very small number of beacons.
\end{abstract}

\section{Introduction}

The theory of identifying codes was proposed in $[4,5]$ as a means of performing robust location detection in emergency sensor networks. That work was motivated by the emergence of wireless tiny sensors $[1,3]$ and the need for emergency personnel to identify the locations of personnel and trapped victims during emergency situations. The key to the approach involved positioning sensors in such a way that overlapping coverage regions are covered by a unique, and hence identifying, set of sensors. It turns out that determining an optimal placement of sensors is equivalent to constructing an optimal identifying code. The work in $[4,5]$ presented an algorithm ID-CODE for generating irreducible identifying codes over arbitrary topologies and proved various properties of this algorithm.

In this work, we describe the experimental design, implementation, and evaluation of the proposed location detection scheme, elaborating on a short summary that appeared in [5]. We provide a detailed description of our experimental framework and analyze various trade-offs associated with its design. Our testbed is implemented on the fourth floor of a nine-story building using wireless-equipped computer laptops. The testbed provides location detection capabilities for a portion of floor space, proving the feasibility of our system. We discuss and provide 
solutions for significant practical issues that arise in implementing such a system. Using extensive data collected for our testbed, we demonstrate the ability of the proposed system to detect user location, and we propose directions for enhancing the system's performance.

The main challenge in the system design is to construct a stable, distinguishable, underlying graph based on wireless connections in order to determine the beacon placement. Part of this challenge involves translating the soft information on node connectivity into a hard binary decision, for the purposes of generating an identifying graph. As part of experimentally determining how to produce this transformation, we have studied the effects of various design parameters, such as transmission power, data rate, packet size, and packet transmission rate. These experiments enabled us to set a packet reception rate threshold for making hard decisions on node connectivity. We present numerical results to illustrate the relationship between our design parameters and the rate of received packets.

The rest of this paper is organized as follows. Section 2 briefly explained related work in the fields of location detection technologies and identifying codes. In Section 3, we describe the outline of our proposed system, explaining the relationship between location detection in wireless networks and the construction of identifying codes for arbitrary graphs. The main theme of our paper is described in Sections 4- 7. The first two of these sections identify a number of design and implementation challenges for our work, and the last two sections describe our experimental wireless testbed, some decoding methods, and an analysis of our results. Finally, our conclusion summarizes the main findings of this work and presents plans for future research.

\section{$2 \quad$ Related Work}

Location detection systems have been proposed and implemented in the literature for a variety of applications. For outdoor applications, the satellite based Global Positioning System (GPS) is the most widely deployed scheme [6]. GPS relies on trilateration of position and time among four satellites, and can determine location in many cases within 1 to 5 meters when used in conjunction with a Wide Area Augmentation System (WAAS). However, signals from the GPS satellite system cannot always penetrate through walls and metallic structures, and therefore GPS reception is often intermittent in indoor settings. Alternative schemes must therefore be implemented for providing reliable location detection in indoor environments.

The Active Badge location system [7,8] was one of the first prototypes of indoor location detection systems. In this system, each user wears a badge that periodically emits a unique ID signal using infrared. This signal is typically received by one of the several receivers scattered throughout a building and forwarded to a central server. The identity of the receiver that overhears the user's signal determines the location of the user. This approach is referred to as proximity-based location detection, since the location of a user is resolved to be that of its nearest receiver. 
The Active Bat [9] and MIT's Cricket [10] systems use ultrasound to provide location detection based on proximity. These systems measure the time-of-flight of ultrasound signals with respect to reference RF signals, thereby allowing a system to calculate the location of a user using a trilateration computation. Active Bat claims an accuracy within $9 \mathrm{~cm}$ for $95 \%$ of the measurements.

The main problem with infrared and ultrasound proximity-based schemes is that their coverage may be lost as soon as a path from a user to its nearby receiver becomes obstructed. This could be due to structural changes (e.g, the opening or closing of a door) or the presence of smoke in a building in fire.

Another powerful approach for indoor location detection is RF (Radio Frequency) because of its inherent ability to penetrate many types of surfaces, especially at lower frequencies. In addition, RF-based indoor location detection has significant cost and maintenance benefits, as it can be readily deployed over existing radio infrastructures, such as IEEE 802.11 wireless LANs. Several RF-based location detection systems have been proposed in the literature. In particular, the RADAR $[12,13]$ system is based on the construction of a Signal to Noise Ratio (SNR) map for a locatable area. The vector of signal strengths received at various base-stations is compared to this map to determine the position of a user. The Nibble system uses a similar approach but, to improve performance, also incorporates a Bayesian model for predicting the likely origin of a signal based on signal quality observed at access points [14].

In this work, we resort to RF to achieve spatial diversity. However, we do not rely on the knowledge of an SNR map, since in many applications, such as disaster recovery, it is impossible to determine an accurate signal map of the environment. Therefore our approach deliberately uses very limited knowledge of the signal landscape so as to be robust to changes. More specifically, its fundamental idea is to build redundancy into the system in such a way that each resolvable position is covered by a unique set of beacons which serve as a positional signature. This approach can be formalized using the theory of identifying codes $[16,17,20]$, and its theoretical foundations are described in detail in $[4,5]$.

\section{System Overview}

In this section, we briefly review our location detection scheme based on the theory of identifying codes $[4,5]$. Our proposed system divides the coverage area into locatable regions, and reports a point in this region as the location for a given target. The system is designed as follows:

- First, a set of points is selected for a given area.

- Then, based on the RF connectivity between the points, beacons are placed and activated on a subset of these points determined by a corresponding identifying code. This activation guarantees that each point is covered by a unique set of beacons.

- An observer can determine its location from the unique collection of ID packets that it receives. 
The following example illustrates the approach. Let the selected points, on a given floor, be $P=\{a, b, c, d, e, f, g\}$, as illustrated in Step 1 in Figure 1(a), and let the RF-connectivity among these points be represented by the arrows in Step 2 Figure 1(b); in other words, there is an arrow between two positions if and only if RF communication is possible between these points. Using the ID-CODE algorithm described in $[4,5]$, we place four beacons at positions $a, d, e$ and $g$. Each beacon periodically broadcasts a unique ID. The user stays for a (small) time $T$ at its current position and collects the ID's from the packets it receives. For instance, in Step 3 Figure 1(c), an observer in the region of point $b$ receives ID's from the beacons at position $a$ and $d$. The set of ID's received at a given position $x$ is called the identifying set of $x$ and denoted $I D(x)$. Since the identifying set of each point in $P$ is unique (see Figure $1(\mathrm{~d})$ ), the user can resolve its location unambiguously using a look-up table. A set of beacons that results into unique identifying sets is called an identifying code.

(a)

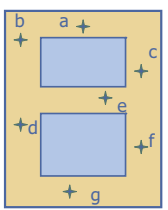

(b)

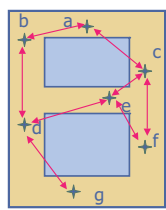

(c)

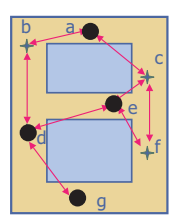

(d)

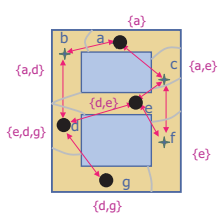

Fig. 1. Our location detection system. (a) Discrete locations. (b) Connectivity. (c) Beacon placements. (d)Covering regions of each discrete point and corresponding identifying sets

\section{The Challenge: Constructing Underlying Graph Based on Wireless Connections}

In our system, a graph representation of the network is essential to determine beacon placements. A node is represented by a circle, and a link between two nodes is usually represented by a line drawn between the nodes. Physically, the connectivity between two nodes implies a medium (such as air, wire, or fiber) that provides the communications system with a channel resource (such as bandwidth) and a system architecture that implements a channel signaling and modulation scheme using the channel resource available in the medium.

However, the wireless medium introduces difficulties for communication by virtue of its inherent nature. In following subsections, we discuss about several issues involving the graph construction in RF networks in detail. We will start with explaining why the connectivity between two nodes is not binary in nature for wireless networks.

\subsection{Gray Zone}

First let us consider radio propagation in free space [22, 23], in which the influence of obstacles is neglected. In this case, path loss becomes the most important 
parameter predicted by large-scale propagation models. We consider the model to be isotropic, where the transmitting antenna radiate power $P_{t}$ uniformly in all directions. Examining path loss will indicate the amount of power available at the receiving antenna of area $A_{e}$ at a distance $r$ meters from the source. Using this model would allow us to easily determine the connectivity status $(\mathrm{ON} / \mathrm{OFF})$ between any two nodes. For a fixed distance $r$, if the power level at the receiver is greater than the power level threshold of the receiving antenna, which is prescribed by manufacturers, the connection is maintained. Otherwise both are disconnected.

To be more realistic, however, we have to consider the propagation in the presence of obstacles (such as ground, walls, or ceilings). There are three main propagation mechanisms that determine and describe path loss [22, 23] as follows: refection, diffraction, and diffusion. These three phenomenons introduce specific geographic location, called gray zone. In the gray zone, the power at the receiving antenna is very low and usually the antenna is not able to correctly detect any packets, but it may occasionally receives a few packets from a source due to multi-path propagation. This leads to a difficulty in making a hard decision about whether any two points in the space are connected. Furthermore, indoor radio propagation is much more variable than the outdoor propagation. The place where antennas are mounted also impacts packet reception. For example, antennas mounted at desk level in a room received vastly different signals from those mounted in the ground or on the ceiling.

\subsection{Environmental Fluctuations}

The channel fading $[22,23]$ occurs when the communicating device and/or components of its environment are in motion. These mobile objects introduce various distinct propagation paths, whose delay differences are very small compared to symbol intervals. The combination of these signals causes the fluctuation of the received signal with time. Furthermore, the speed of propagating waves depends on the temperature, because temperature affects the strength of particle interactions in air. This implies that the number of packets received between a pair of nodes also varies with time. As a result, $\mathrm{RF}$ connectivity is significantly impacted by changes in environment (such as movement of people and objects, changes in temperature, etc.) [24].

\subsection{Orientation Issues}

The radiation pattern of an antenna [25] plays an important role in packet transmission and reception, especially in a dense multi-path environment such as inside buildings. In general, an antenna can either be an omni-directional antenna, or a directional antenna. An omni-directional antenna can radiate or receive equally well in all directions, which implies that all packets are received equally well. On the other hand, a directional antenna can receive best from a specific direction. Thus if the radiation pattern of receiver antenna is directional, it is possible that two points may be connected if the receiver faces one direction, but may not be in other directions for the indoor environment. However, 
using omni-directional antennas may lead to indistinguishably graphs, so the directional antenna is useful for designing the desirable system in our scheme by adding or removing some links. Furthermore, the body of person carrying the receiver may absorb or block the packets in particular orientation, which introduces a systematic source of error.

\subsection{Best Decision Formulation}

Questions often arise concerning the meaning of there being a connection between any two points. Thus, making decisions about connectivity that minimize the probability of a wrong decision is very important in our location detection scheme. An usual strategy, such as Bayesian formulation [26], is not suitable because its decisions are based on the ground truth, which is inherently absent in our proposed system. The connection between any pair of nodes is not well defined for example, receiving one out of forty packets per second from a source may be considered "connected" in a system because the receiver can communicate to the source. On the other hand, one also can decide that it is "disconnected" because the receiver receives very small number of packets compare to the number of packets that actually are transmitted from the source. Therefore, finding a proper decision formulation to determine whether two nodes are connected is very important for improving the performance of the system.

\section{$5 \quad$ Design Parameters}

One important step prior to implementing our location detection system is to understand the relationship between the design parameters and the connectivity. Note that in our experiment, we used packet reception rate as a key metric for determining connections between any two points. We have built a small testbed to illustrate how to set the adjustable design parameters of the system in such a way that the connectivity between any two nodes can be clearly defined.

The testbed consists of five office rooms. Each room has a dimension of 9.5 feet $\times 18$ feet. Rooms are separated by walls with an attenuation factor of $3 \mathrm{~dB}$ (measured at $1 \mathrm{~mW}$ transmitting power). We deployed two computer laptops serving as a transmitter and a receiver, both running Red Hat 8.0 and equipped with a Cisco 350 series wireless $802.11 \mathrm{~b}$ adapter [27], operating at the $2.4 \mathrm{GHz}$ band. During the experiment, we observed that the radiation pattern of this adapter is directional. Thus the orientation of the transmitter's antenna and the receiver's antenna are fixed throughout this experiment. The transmitter broadcasted user datagram protocol (UDP) [21] packets at a rate of 40 packets per second. We determine packet reception range by placing the transmitter in the leftmost room and closed to the leftmost wall, moving the receiver away from the transmitter and measuring the number of packet received for 5 minutes at position closest to the other wall. We increased number of walls and the distance between receiver and transmitter by moving receiver into the next room. Thus the packet reception range is represented by the minimum number of walls (or rooms) that makes the receiver disconnected from the transmitter. In our system, 
we are seeking for the setting such that the number of received packets in front of a wall and behind that wall are much different. In other words, the physical distance to transit from well connected state to disconnected state should be minimized. There are four parameters that we are interested in: data rates, transmitting power, packet size and packet arrival rate distribution.

- Data Rates: Cisco Aironet 350 Client Adapters support 1, 2, 5.5 and 11 Mbps data rates [27]. We chose to compare results between $1 \mathrm{Mbps}$ and 11 Mbps and transmitted packets with length of 1000 bytes and transmitting power of $1 \mathrm{~mW}$. Figure 2(a) illustrates that the packet reception range of a transmitter with $1 \mathrm{Mbps}$ data rate is larger than the range of transmitter with $11 \mathrm{Mbps}$. The number of packets received from the 1-Mbps transmitter gracefully decreases when the distance increases, which is undesirable for our proposed system. Thus high data rate can provide sharper range to determine whether two nodes are connected.

- Transmitting Power: Increasing transmitting power also increases transmission range. For a Cisco Aironet 350 Client Adapter, the transmission power is varied from $1 \mathrm{~mW}$ to $100 \mathrm{~mW}$ [27]. In our experiment, we wanted to compare between $1 \mathrm{~mW}$ and $100 \mathrm{~mW}$ transmitting power. The transmitter transmitted packets with length 1000 bytes at $11 \mathrm{Mbps}$ data rate. In

(a)

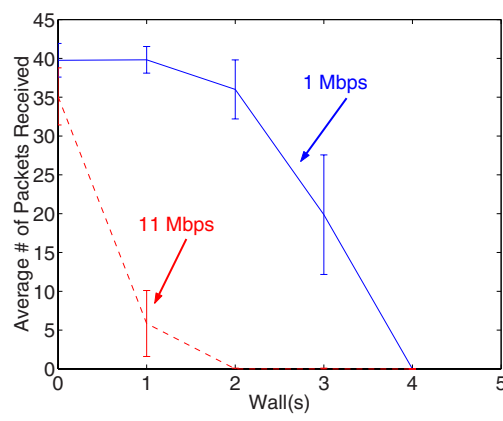

(c)

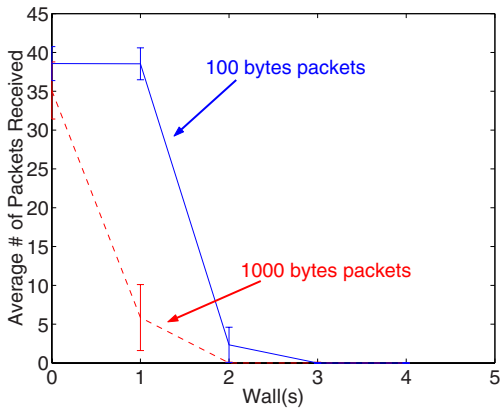

(b)

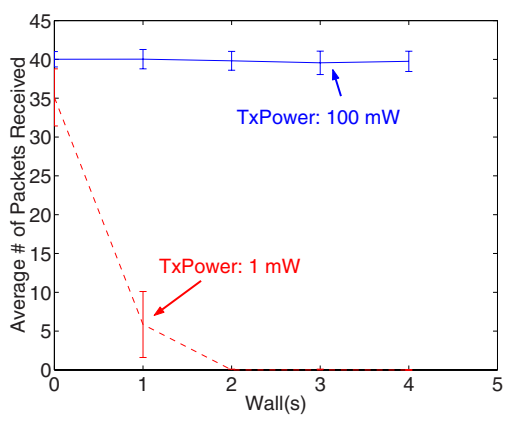

(d)

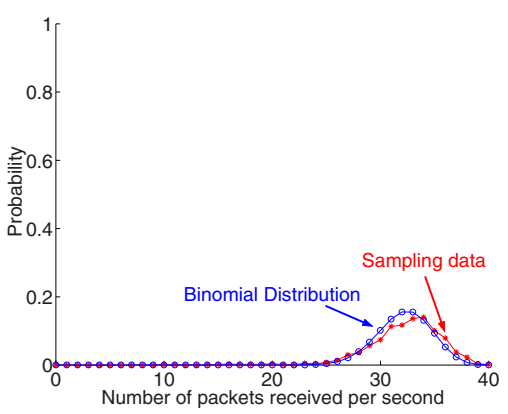

Fig. 2. Reception Range versus (a)data rates, (b)transmitting power, (c)packet size and (d)packet arrival rate distribution 
Figure 2(b), packets transmitted with $1 \mathrm{~mW}$ power could not go through the second wall whereas packets of $100 \mathrm{~mW}$ were received well even in the fifth room. Therefore, the transmitting power can be used to control the coverage area of a transmitter.

- Packet Size: In the binary symmetric channel, each bit will be received correctly with probability (1-p) and incorrectly with probability $\mathrm{p}$, where $\mathrm{p}$ is the probability of error due to noise in the channel. Intuitively, a larger packet has a higher probability of being corrupted. Thus the packet reception range of large packets should be small. In our experiment, we transmitted 100-byte packets and 1000-byte packets, respectively, at the data rate of $11 \mathrm{Mbps}$ and $1 \mathrm{~mW}$ transmitting power. Figure 2(c) clearly depicts that transmitting larger packets provides a smaller range, as expected.

- Packet Arrival Rate Distribution: We also can model a packet as a symbol in the binary symmetric channel, where each packet will be correctly detected with probability (1-p). The connection between any two positions in the network can be represented by a value of p. Given a fixed packet transmission rate $n$, the reception of each packet has two possible outcome, "success" and "failure". All packets are independent, so the outcome of a packet has no effect on the outcome of another. The probability of success is (1-p). These indicate that the packet arrival rates should follows a binomial distribution. As expected, we empirically have found that the distribution of packet arrival rates are Binomially Distributed, as shown in Figure 2(d). Thus value of $\mathrm{p}$ for each connection can be observed based on the average number of packet received.

\section{$6 \quad$ Experimental Testbed}

\subsection{Testbed Description}

Our testbed was located on the 4th floor of the nine-story building. The floorplan is shown in Figure 6. There were 10 positions in the test-bed, which are to be located. Pentium-based laptop computers running Red Hat Linux 8.0 and equipped with an IEEE 802.11b standard compliant Cisco Aironet 350 series Client Adapter [27] were used in this experiment. As we discussed earlier in Section 5, we observed that transmitting packets at high data rates provided a sharp packet reception range. On the other hand, increasing transmit power increased the size of reception range. Furthermore, we also found that increasing packet size will decrease the size of the gray zone. Thus, in this testbed each transmitter transmits $40 \mathrm{UDP}$ packets per second at a data rate of $5.5 \mathrm{Mbps}$ and transmission power of $100 \mathrm{~mW}$. Each packet is 1000-byte long including the transmitter's ID. This setting was obtained by measuring connectivity inside the testbed to obtain the stable distinguishable underlying graph.

The connectivity between every pair of positions was determined as follows: a transmitter was placed at one of the points and a receiver was placed at another point (as marked in Figure 6). Then, the transmitter was set to broadcast 40 packets per second, each containing the transmitter ID. If the receiver received 
at least 20 packets per second in average for five minutes each for all antenna orientations, the position were considered to be connected. This model of connectivity was used in this experiment for simplicity. The connectivity between the points is observed to be reliable with the setup used in this test-bed. The underlying graph was built using the connectivity information between every pair of points obtained as above. In our next step, we applied the ID-code algorithm to determine transmitter placements. The result from the algorithm suggested us to place four transmitters at position 0 , position 1 , position 2 and position 3 as indicated in Figure 6. The identifying set at each position is given in Figure 3.

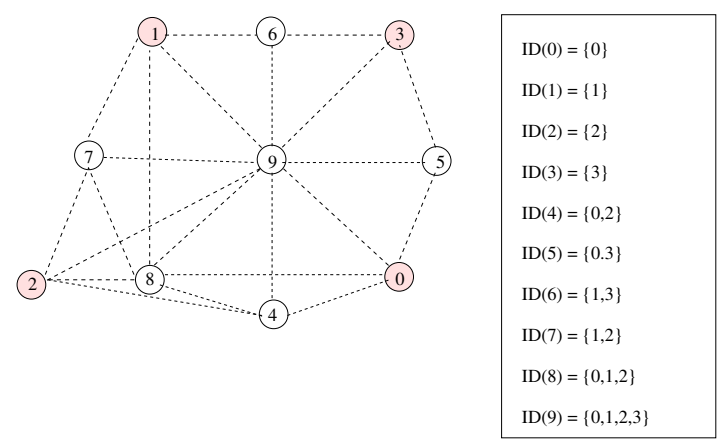

Fig. 3. The connectivity graph of the testbed and its identifying codes. The filled dots denote the positions that transmitters are placed

\subsection{Data Collection and Processing}

The data collection phase is an important step in our research methodology. We wrote a Java application to control the data collection process at the receiver. The packet arrival rate from all transmitters at each position was recorded. The information was collected during the day, when there are other possible sources of fluctuation such as moving people and objects. We observed that the packet arrival rate also varies with the receiver's orientation. Therefore, we also recorded the direction (North, South, East, West) that the wireless adapter is facing at the time of measurement.

The data collection phase is divided into 2 parts as follows:

- Part I: The Distribution of Packet Arrival Rate. In this test-bed, we are interested in determining the correctness of the system by measuring the probability of error in locating the region in which a stationary user is located. We have collected the data over extended periods (at least one hour per position) to observe the distribution of the packet arrival rate. For each orientation of each position, we recorded 1000 samples.

- Part II: The Coverage Region. Our location detection scheme discretizes the space. Therefore, one important characterization of the test-bed is the extent of the region around a reference position, where the receiver receives 
the same code. For this purpose, the floor is divided into small grid points and the receiver is placed on one of the grid points for 60 samplings per orientation.

\section{$7 \quad$ Experimental Results and Analysis}

\subsection{Packet Rate Distributions}

In our testbed, we make use of packet arrival rates to determine the connectivity between any two points and then decode the position. To demonstrate that the packet arrival rate is a reasonable measure of connectivity, we show some examples of the probability distribution of the packet arrival rate for locations in our test-bed shown in Figure 4. The gap between codewords zero and one are sharply separated in almost all positions. This indicates that using packet arrival rates to infer location codewords is a promising approach for our scheme.
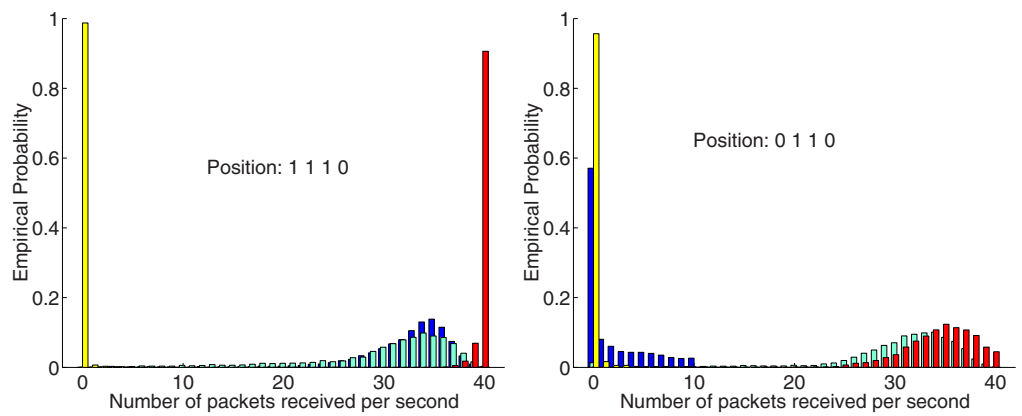

Fig. 4. The probability of packet arrival rate recorded at the five reference positions at transmission rate 40 packets/sec at position 1110 and 0110

\subsection{Finding the Best Decoding Threshold}

In our basic approach, given sample packet arrivals from each transmitter, called an observed packet vector $\left(n_{0}, n_{1}, n_{2}, n_{3}\right)$, we determine the location that best matches this observation to a corresponding binary codeword in the lookup table. The lookup table is created when the system is setup and contains codewords corresponding to each location in the area. If a user obtains an unknown codeword, the system will choose the closest codeword as user's location. In our experiment, we use thresholding scheme, which is one of the simplest methods to decode an observed packet vector. If the sample interval exceeds a certain threshold $\theta$, it will set to $1 \mathrm{~s}$ otherwise $0 \mathrm{~s}$. To find the best threshold, the system needs to be trained by samples during the setup phase. This is a workable strategy, but it may not be optimal. You can find other possible decoding techniques in [2].

We use the empirical data obtained in the data collection and processing phase part I (see Section 6.2) to find the threshold that minimize probability of 
decoding errors. We characterize the performance of our estimate of a stationary user's location using probability of error to determine the region in which the user is located. In the analysis, we use all of the $5^{*} 4000$ samples collected for all combinations of user locations and orientation (5 locations and 1,000 samples per location per orientation). For 1,000 iterations, we randomly chose 400 samples from each location regardless of the orientation as the training data set in the off-line phase and the remaining data is the testing data set in the online phase. In each iteration, we use the training data set to determine the best threshold that minimizes the overall probability of error for the whole system. We then apply this threshold to the testing data set, count the number of samples that are incorrectly decoded and compute probability of error. Finally we determine the average of the probabilities of error from all iterations to represent the performance of the single threshold method.

Figure 5 shows that 15 is the best empirical threshold of decoding for this testbed. Considering probability of error for varying threshold, we found that the threshold that gives the minimum probability of error is approximately between 10 and 20 . We observe that increasing the training data set slightly improves the performance of the system [2].
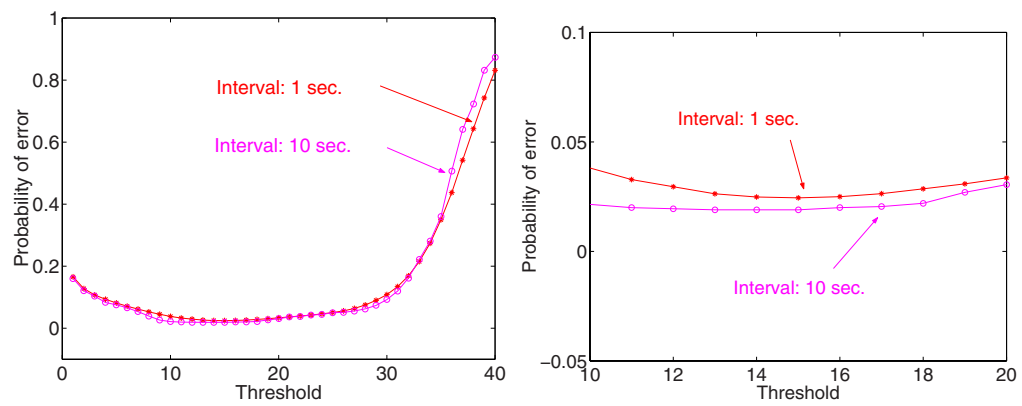

Fig. 5. The probability of error for varying threshold with Data Collecting Interval size of $1 \mathrm{sec}$ and $10 \mathrm{sec}$

\subsection{Data Collecting Interval}

In wireless networks, changes in environment can cause fluctuation in the number of packets received. Collecting packets longer may help users to observe the real channel state and obtain the correct codeword. To analyze this, we compare the interval time to collect data per one decoding step between 1 second and 10 seconds. To decode the current position, we average all samples which are collected in the given interval. We then decode by the threshold and find the best matched location. Figure 5 explains the performance of the system when we increase the data collecting interval. As expected, most of thresholds show that the larger interval can reduce the probability of error in decoding step. However, when the threshold was set very high, the longer interval significantly degraded the performance of the system due to lost packets. 


\subsection{Impact of Packet Transmission Rate}

Intuitively, transmitting packets at high packet rate can separate the packet arrival rate of connected transmitters and disconnected transmitters better than the low packet rate. However, if the current location of a receiver is identified by more than one transmitter, the number of packets received depends on the current network traffic. To observe the impact of packet transmission rate, we conducted our experiments at a position whose corresponding codeword is 110 . All three transmitters periodically emit ID packets with the same packet transmission rates $(5,10,40,60$, and 70 packets/sec, respectively).

Figure 6 (a) clearly shows that at the interested position, very low packet transmission rate (such as 5 packets/sec and 10 packets/sec) cannot provide clear separation between codeword one and codeword zero. Since packets were periodically transmitted, low data rate creates large interval between two consecutive packets, which implies that at the receiver, the probability of packet collisions occurring is low. The gap between the distribution of the third transmitter whose codeword is zero and the others (codeword one) increased when the packet rate increased. With a higher data rate, the receiver can receive more packets from transmitters who are connected to the position. The probability of packet collisions is high enough to eliminate some spurious packets from the third transmitter. However, when the packet transmission rate is greater than 60 packets/second, packet collisions result in less number of packets being received. Consequently, the difference between codeword one and codeword zero based on the number of packets is decreased.

Furthermore, since we propose to use beacons for broadcasting identified packets. Thus transmitting packets at very high rate creates high power consumption which is inefficient. In order to obtain the best performance, one needs to find the optimal packets transmission rate that minimizes the probability of error in a system.

(a)

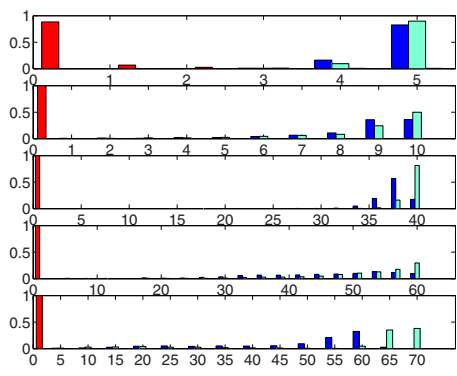

(b)

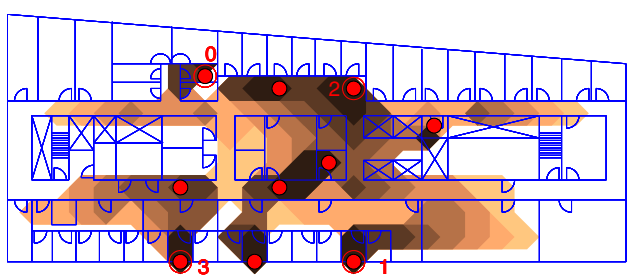

Fig. 6. (a) Setting a good packet transmission rate can improve the performance of the system (b) A diagram demonstrates the range of each reference position with the confident level of decoding at least $70 \%$ 


\subsection{Coverage Region}

To evaluate the goodness of our system, another characteristic that we consider is the coverage region of the selected points, in other words, the resolution. Resolution is the distance between the actual user's location and the decoded location. The contour map in Figure 6(b) shows the resolution (0-70 feet) of the system with a $70 \%$ confidence level. Deploying four transmitters, the floor space can be divided into ten regions. These results show that an id code-based location detection system can achieve good accuracy with a very few number of beacons.

\section{Conclusion}

We have developed, analyzed, and refined a comprehensive set of techniques for indoor location detection systems based on the theory of identifying codes. In our scheme, based on the theory proposed in $[4,5]$, beacons are positioned in such a way that overlapping coverage regions are covered by a unique, and hence identifying, set of beacons.

The main contribution of our work is the development of a prototype location detection testbed in an office building using commodity Wi-Fi hardware. Our goal for this prototype was to provide reasonably accurate location detection capabilities for a portion of the floor-space of this building. Our experimental results show that our location detection scheme is feasible and useful. With four transmitters, our system can provide ten distinct locatable regions on the fourth floor of our chosen office building. Our system was able to identify a user's location region with a high degree of accuracy by using a simple thresholding scheme for determining RF connectivity.

Nevertheless, there remain a number of open issues. For one, it would be interesting to investigate whether signal attenuation and modulation can be exploited to enable more stable connections in an indoor environment. On the other hand, we plan to investigate the use of different carrier signals to avoid the absorption problems that are typical for the frequencies used by 802.11. Finally, the threshold decoder we used is very simple, and more sophisticated soft-decision decoders can very likely improve the performance of the system.

\section{Acknowledgements}

The authors would like to thank Dr. M. Karpovsky and Dr. L. Levitin for introducing them to identifying codes, S. Ray and F. De Pellegrini for fruitful discussions while developing the ID-CODE algorithm, Dr. J. Carruthers for his advice and C. Malladi for experiments on the testbed.

This work was supported in part by the US National Science Foundation under grants CAREER ANI-0132802, CAREER CCR-0133521, and ANI-0240333. 


\section{References}

1. S. E.-A. Hollar, "COTS dust," Master's thesis, University of California, Berkeley, 2000.

2. U. Rachanee, "Location Detection System in Emergency Sensor Networks Using Robust Identifying Codes ," Master's thesis, Boston University, Boston, 2003.

3. J. M. Kahn, R. H. Katz, and K. S. J. Pister, "Next century challenges: mobile networking for "smart dust'," in Proceedings of the fifth Annual ACM/IEEE international conference on Mobile computing and networking, (Seattle, WA, United States), ACM, 1999.

4. S. Ray, R. Ungrangsi, F. De Pellegrini, A. Trachtenberg, and D. Starobinski, "Robust location detection in emergency sensor networks," in IEEE INFOCOM 2003, (San Francisco, CA), IEEE, 2003.

5. Saikat Ray, David Starobinski, Ari Trachtenberg and Rachanee Ungrangsi, "Robust Location Detection with Sensor Networks," IEEE JSAC (Special Issue on Fundamental Performance Limits of Wireless Sensor Networks), vol. 22, No.6, August 2004 .

6. US Coast Guard Navigation Center, Global Positioning System Signal Specigication. 2nd edition ed., 1995.

7. R. Want, A. Hopper, V. Falcao, and J. Gibbons, "The active badge location system," ACM Transactions on Information Systems, vol. 10, pp. 91-102, January 1992.

8. R. Want, B. N. Schilit, N. I. Adams, R. Gold, K. Peterson, D. Goldberg, J. R. Ellis, and M. Weiser, "An overview of the PARCTAB ubiquitous computing experiment," IEEE Personal Communications, vol. 2, pp. 28-43, December 1995.

9. A. Harter, A. Hopper, P. Steggles, A. Ward, and P. Webster, "The anatomy of a context-aware application," in MobiCom'99, ACM, 81999.

10. N. B. Priyantha, A. Chakraborty, and H. Balakrishnan, "The cricket locationsupport system," in 6th ACM International Conference on Mobile Computing and Networking (ACM MOBICOM), (Boston, MA), ACM, 2000.

11. N. B. Priyantha, A. K. L. Miu, H. Balakrishnan, and S. Teller, "The cricket compass for contextaware mobile applications," in 7th ACM Conference on Mobile Computing and Networking (MOBICOM), (Rome, Italy), ACM, July 2001.

12. P. Bahl and V. N. Padmanabhan, "RADAR: An in-building RF-based user location and tracking system," in IEEE INFOCOM 2000, (Tel Aviv, Israel), IEEE, 2000.

13. P. Bahl, A. Balachandran, and V. Padmanabhan, "Enhancements to the RADAR User Location and Tracking System," Tech. Rep. MSR-TR-2000-12, Microsoft Research, February 2000.

14. P. Castro, P. Chiu, T. Kremenek, and R. R. Muntz, "A probabilistic room location service for wireless networked environments," in Ubicomp, (Atlanta, GA), ACM, 2001.

15. N. Bulusu, J. Heidemann, and D. Estrin, "GPS-less low cost outdoor localization for very small devices," in Proceedings of IEEE Personal Communications, pp. 28 34, IEEE, October 2000.

16. M. G. Karpovsky, K. Chakrabarty, and L. B. Levitin, "A new class of codes for identification of vertices in graphs," IEEE Transactions on Information Theory, vol. 44, pp. 599-611, March 1998.

17. I. Charon, O. Hudry, and A. Lobstein, "Identifying codes with small radius in some infinite regular graphs," The Electronic Journal of Combinatorics, vol. 9, 2002. 
18. N. S. V. Rao, "Computational complexity issues in operative diagnosis of GraphBased systems," IEEE Transactions on Computers, vol. 42, pp. 447-457, April 1993.

19. K. Chakrabarty, S. S. Iyengar, H. Qi, and E. Cho, "Grid coverage for surveillance and target location in distributed sensor networks," IEEE Transactions on Computers.

20. K. Chakrabarty, H. Qi, S. S. Iyengar, and E. Cho, "Coding theory framework for target location in distributed sensor networks," in International Symposium on Information Technology: Coding and Computing, pp. $130-134,2001$.

21. L. L. Peterson and B. S. Davie, Computer Networks: A systems approach. Morgan Kaufmann, 2 ed., 2000.

22. Mark and Zhuang, Wireless Communications and Networking. Prentice Hall PTR, 2003.

23. T. S. Rappaport, Wireless Communications: Principles and Practice. Upper Saddle River NJ: Prentice Hall, 1996.

24. H. Hashemi, "The indoor radio propagation channel," in Proceedings of the IEEE, no. 7, pp. 943-968, July 1993.

25. J. C. Liberty, Jr. and T. S. Rappaport, Smart Antennas for Wireless Communications: IS-95 and Third Generation CDMA Applications. Prentice Hall PTR, 1999.

26. H. Chernoff and L. Moses, Elementary Decision Theory. Dover, 2 ed., 1986.

27. http://www.cisco.com/en/US/products/hw/wireless/ps4555/products_datasheet_ 09186a0080088828.html, "Cisco Aironet Wireless LAN Client Adapters Cisco Aironet 350 Series Client Adapters - Cisco Systems." 